\title{
CTA Evaluation of Bioresorbable Scaffolds versus Metallic Coronary Stents - a Feasibility Study
}

\author{
Ioan Ferenț1,2,3, András Mester1,2,3, Monica Chițu1,2,3, Annabella Benedek1,2,3, Mihaela Rațiu1,2, \\ Roxana Hodas ${ }^{1,2,3}$, Imre Benedek ${ }^{1,2,3}$ \\ ${ }^{1}$ University of Medicine and Pharmacy, Tîrgu Mureș, Romania \\ ${ }^{2}$ Center of Advanced Research in Multimodality Cardiac Imaging, Cardio Med Medical Center, Tîrgu Mureș, Romania \\ ${ }^{3}$ Cardiac Critical Care Unit, Clinic of Cardiology, County Clinical Emergency Hospital, Tîrgu Mureș, Romania
}

\section{CORRESPONDENCE}

\section{András Mester}

Str. Gheorghe Marinescu nr. 38

540139 Tîrgu Mureș, Romania

Tel: +40 265215551

E-mail: andras.mester@yahoo.com

\section{ARTICLE HISTORY}

Received: August 4, 2018

Accepted: September 10, 2018
Ioan Ferenț • Str. Gheorghe Marinescu nr. 38, 540139 Tîrgu Mureș, Romania. Tel: +40 265215 551, E-mail: ionutferent2013@gmail.com

Monica Chițu • Str. Gheorghe Marinescu nr. 38 , 540139 Tîrgu Mureș, Romania. Tel: +40 265215551 E-mail: iulia.chitu@yahoo.com

Annabella Benedek • Str. Gheorghe Marinescu nr. 38 540139 Tîrgu Mureș, Romania. Tel: +40 265215 551, E-mail: annabell.benedek@yahoo.com

Mihaela Rațiu • Str. Gheorghe Marinescu nr. 38 540139 Tîrgu Mureș, Romania. Tel: +40 265215551 , E-mail: d_a_mihaela@yahoo.com

Roxana Hodas - Str. Gheorghe Marinescu nr. 38 540139 Tîrgu Mures, Romania. Tel: +40 265215 551, E-mail: roxana.hodas@yahoo.ro

Imre Benedek • Str. Gheorghe Marinescu nr. 38 540139 Tîrgu Mureș, Romania. Tel: +40 265215551 E-mail: imrebenedek@yahoo.com

\begin{abstract}
Background: Computed tomography angiography (CTA) presents important limits in in-stent restenosis (ISR) evaluation in case of metallic coronary stents, due to the artifacts determined by stent struts, which alter in-stent plaque analysis. In case of bioresorbable scaffolds, stent strut resorption allows accurate evaluation of the vessel wall. Aim of the study: This study aims to compare the feasibility of CTA as a follow-up imaging method for ISR diagnosis following elective PTCA procedures, between bioresorbable scaffolds and metallic coronary stents. Material and methods: We conducted a prospective, observational study on 73 patients with elective PTCA procedures in their medical history, in whom 113 stents were assessed via CTA in order to diagnose ISR. Based on stent type, the patients were divided into two groups: Group 1 - patients with bioresorbable vascular scaffolds (BVS) $(n=30)$; and Group 2 - patients with bare metal stents (BMS) $(n=43)$. Plaque analysis was possible only in the BVS group with a post-processing research-dedicated software, Syngo.via Frontier, which identified plaque morphology and virtual histology composition. Results: After CTA evaluation, the BVS group presented a significantly higher incidence of severe coronary artery disease (CAD) (Group $1-73 \%$ vs. Group $2-30 \%$, $p<0.0001)$. The proximal part of the right coronary artery (RCA) presented a significantly higher percentage of metallic stents ( $14 \%$ BMS vs. $2 \%$ BVS, $p=0.0029$ ). The comparative analysis of CTA sensibility for the visual evaluation of ISR identified a significantly higher percentage of diagnostic CT evaluations in the BVS group (Group $1-94 \%$ vs. Group $2-76.19 \%, p=0.0006$ ). CTA evaluation provided the most accurate results for the 3.0 and $3.5 \mathrm{~mm}$ devices. Regarding CTA sensibility for ISR diagnosis, the BVS group presented the smallest incidence of non-diagnostic CT evaluations. Conclusions: CTA evaluation of bioresorbable scaffolds is superior to metallic stent assessment, the latter being influenced by numerous sources of error dependent mainly on the presence of the metal structure.
\end{abstract}

Keywords: CTA, in-stent restenosis, bioresorbable scaffolds, metallic coronary stent 


\section{INTRODUCTION}

In the last decades, percutaneous coronary angioplasty has become one of the most common interventions for the treatment of coronary artery disease (CAD). ${ }^{1}$ However, restenosis, occuring at the site of treated injuries, and the possible need for repeated revascularization are the main drawbacks encountered in interventional cardiology. ${ }^{2} \mathrm{Re}-$ stenosis represents a complex phenomenon that starts immediately after vessel barotrauma, induced by coronary angioplasty. ${ }^{3-5}$ Based on the moment of occurrence, instent restenosis (ISR) can be classified in early ISR - due to elastic recoil and in-lumen axial plaque relocation, characterizes balloon angioplasty primarily, and late ISR - the consequence of thrombus reorganization, neointima formation, vascular remodeling, and resolution of the inflammatory process.

The need for adequate follow-up of patients undergoing coronary angioplasty emerged since the first angioplasty, performed more than 40 years ago. ${ }^{2}$ After percutaneous coronary intervention (PCI), the patient falls into the category of patients that must be followed regularly, being at risk for stent occlusion by thrombosis or restenosis. ${ }^{6}$ The complete assessment of the type of occlusion is essential in order to choose the optimal revascularization strategy (DES vs. DEB). Thus, choosing an optimal imaging method to monitor stent permeability represents a priority in interventional cardiology. Identifying new, modern imaging techniques that can be applied as follow-up methods of implanted scaffolds represents an interest in interventional cardiology, knowing that the early identification of a significant restenosis simplifies the subsequent revascularization procedure. ${ }^{7}$

The CTA evaluation of metallic stents is based on contrast agent attenuation around the area of neointimal hyperplasia. However, this process presents major limitations due to metallic stent struts, which lead to significant artifacts and misinterpretations. Regarding post-implantation follow-up, bioresorbable scaffolds present the advantage of accurate evaluation via CTA in terms of luminal dimensions and plaque analysis. The accuracy and feasibility of multislice CTA in the evaluation of bioresorbable scaffolds, assessed in a multicentric study, may consolidate noninvasive imaging techniques as reference methods in this type of patient follow-up. ${ }^{8,9}$

CTA evaluation has become one of the most popular imaging techniques in cardiology, its relevance in vulnerable coronary plaque evaluation being widely accepted. ${ }^{10-14}$ In a recent study, conducted on 238 patients enrolled in the ABSORB II trial with 258 coronary ath- erosclerotic lesions treated with bioresorbable scaffolds, subjects were evaluated via invasive angiography, intravascular ultrasound (IVUS), and CTA at the 3-year follow-up after PCI. After analyzing the results, CTA proved to have a similar diagnostic precision to conventional angiography in terms of identifying and quantifying the severity of ISR. ${ }^{15}$

In this regard, the accuracy of CTA evaluation represents another advantage of bioresorbable scaffolds in the interventional treatment of coronary atherosclerotic lesions.

\section{OBJECTIVES}

CTA presents important limitations in ISR evaluation in case of metallic coronary stents due to the artifacts determined by stent struts, which alter in-stent plaque analysis.

In case of bioresorbable scaffolds, stent strut resorption allows the accurate evaluation of the vessel wall. This study aims to analyze the utility of CTA in the follow-up evaluation of bioresorbable scaffolds versus metallic coronary stents, in order to diagnose ISR after PCI.

\section{MATERIAL AND METHODS}

We conducted a prospective, observational study, which included 73 patients, treated by implanting one or more stents for coronary artery disease, in the CardioMed Medical Center Tîrgu Mureș, according to ESC guidelines, between January 2015 and March 2017. Based on stent type, the study subjects were divided into two groups: Group 1 - 30 patients with 50 implanted bioresorbable scaffolds (ABSORB 1.1/Abbott Vascular) and Group 2 - 43 patients with 63 metallic (MULTI-LINK ULTRA/Abbot Vascular, Tsunami Gold/Terumo, Guidant MULTI-LINK PLUS/ Abbot Vascular) and drug-eluting stents (Resolute Integrity/Medtronic, XIENCE/Abbot Vascular).

Patients with PTCA and stent placement in their medical history were included in this study. Other inclusion criteria were age over 18 years, BMI $<40 \mathrm{~kg} / \mathrm{m}^{2}$, signed written informed consent. Exclusion criteria were STelevation myocardial infarction or non-ST-elevation myocardial infarction as diagnosis of PCI indication, electric and hemodynamic instability at admission, allergy to iodine contrast material, indication for oral anticoagulation therapy à la longue, acute renal failure or terminal-stage chronic kidney disease, pregnancy or lactation, active malignancy/end-stage disease with life expectancy under 1 year, or refusal to provide written informed consent for study enrollment. 
Each patient underwent invasive angiography and CTA evaluation, between 12 and 24 months after PTCA, in order to evaluate the stent's degree of permeability. After PTCA, each subject performed the 1-, 6-, 12-, and 18-month follow-up visit with major adverse cardiovascular events (MACE) rate evaluation.

\section{CT scanning protocol}

Each CTA evaluation was performed using a 64- and 128-slice dual-source CT scanner (SOMATOM Definition, Siemens Healthcare) available in the Laboratory of Advanced Research in Cardiac Multimodal Imaging of the Cardio Med Medical Center of Tîrgu Mureş, with 64 detector rows $\times 0.5 \mathrm{~mm}$ and $128 \times 0.6 \mathrm{~mm}$ respectively, $330 \mathrm{~ms}$ rotation time, and a table feed of $0.2-0.4 \mathrm{~mm}$ per rotation.

The scanning protocol involved a $4-6 \mathrm{~h}$ fasting period, avoidance of smoking, caffeine use, and intense physical exercises $24 \mathrm{~h}$ prior to the CT examination; a heart rate below 60 bpm during image acquisition, using a bradycardization protocol with Metoprolol 25-50 mg or Ivabradine $5 \mathrm{mg}$ orally, $1 \mathrm{~h}$ prior to the CT scan. The scanning protocol was explained to each patient, the study subjects being instructed to follow audio instructions regarding respiration. The Ultravist $370 \mathrm{mg} \mathrm{I} / \mathrm{ml}$ contrast agent (Bayer Healthcare, Germany) was administered through a peripheral venous catheter (16-22 G) placed in the antecubital vein, followed by $50 \mathrm{ml}$ of $0.9 \% \mathrm{NaCl}$ solution. The dose of the contrast agent was adjusted according to body weight and scan time, based on the formula: contrast agent volume $(\mathrm{mL})=($ scan time +10$) \times$ preset flow rate, at a flow rate of $5 \mathrm{~mL} / \mathrm{s}$, a tube voltage of $120 \mathrm{kV}$, and a tube current of $400 \mathrm{mAs}$.

The CT scanning protocol included a topography in antero-posterior and lateral incidence, in order to frame the acquisition territory from the base of the sternum to the apex of the heart. "Automatic bolus tracking" technique was used to detect the contrast reaching the descending aorta. The region of interest was placed in the descending aorta and the median region of the heart, and image acquisition was performed after the audio command of breathing, at 10 seconds after the beginning of contrast material injection.

\section{CT image reconstruction and data analysis}

Post-processing of CT acquisitions was performed using a dedicated software, Syngo.via Frontier with a Siemens workstation (Siemens AG, Erlangen, Germany) and QCTA RE (Medis, Leiden, Holland).
In order to reconstruct images of the coronary arteries, cardiac cycles with minimal movement or frequency artefacts were selected. Stents were evaluated via curved multiplanar reconstructions, defining the following imagistic patterns:

- permeable stent without signs of neointimal hyperplasia: homogeneous opacification of the vascular lumen;

- permeable stent with insignificant neointimal hyperplasia: longitudinal attenuation of the contrast agent, with a lumen reduction $<50 \%$;

- significant ISR: longitudinal and transverse attenuation of the contrast agent, with a lumen reduction $>50 \%$;

- ISR with occlusion: complete lack of lumen opacity.

The analyzed stent was considered correctly evaluated if its lumen was visible in all its length and contrast attenuation was not significantly altered by artifacts caused by metals struts or motion. In the situation of getting a low-quality, blurry and unequally acquired image with a series of artifacts, the CT evaluation was considered nondiagnostic. Together with the stented coronary artery segment, $5 \mathrm{~mm}$ before and after the scaffold were evaluated with maximum intensity projection in order to define restenosis in the stent ends.

\section{Statistical analysis}

Statistical analysis was performed using GraphPad Prism 7 software (GraphPad Software, Inc., San Diego, USA). A two-tailed $p$ value of $<0.05$ was considered statistically significant. The D'Agostino Pearson normality test was used to test the normality of distribution of numerical data, continuous data was shown as mean \pm standard deviation and median respectively, and categorical variables were expressed as percentages and integer values. Pearson and Spearman coefficients were used for correlation analysis.

\section{Ethics}

This study was conducted according to the current revised edition of the Declaration of Helsinki. The study was performed under the approval of the Ethics Committee of the University of Medicine and Pharmacy of Tîrgu Mureș (approval no. 338/17.11.2017) and the Ethics Committee of the CardioMed Medical Center (approval no. 29/28.12.2017). Written informed consent was obtained from each patient. 


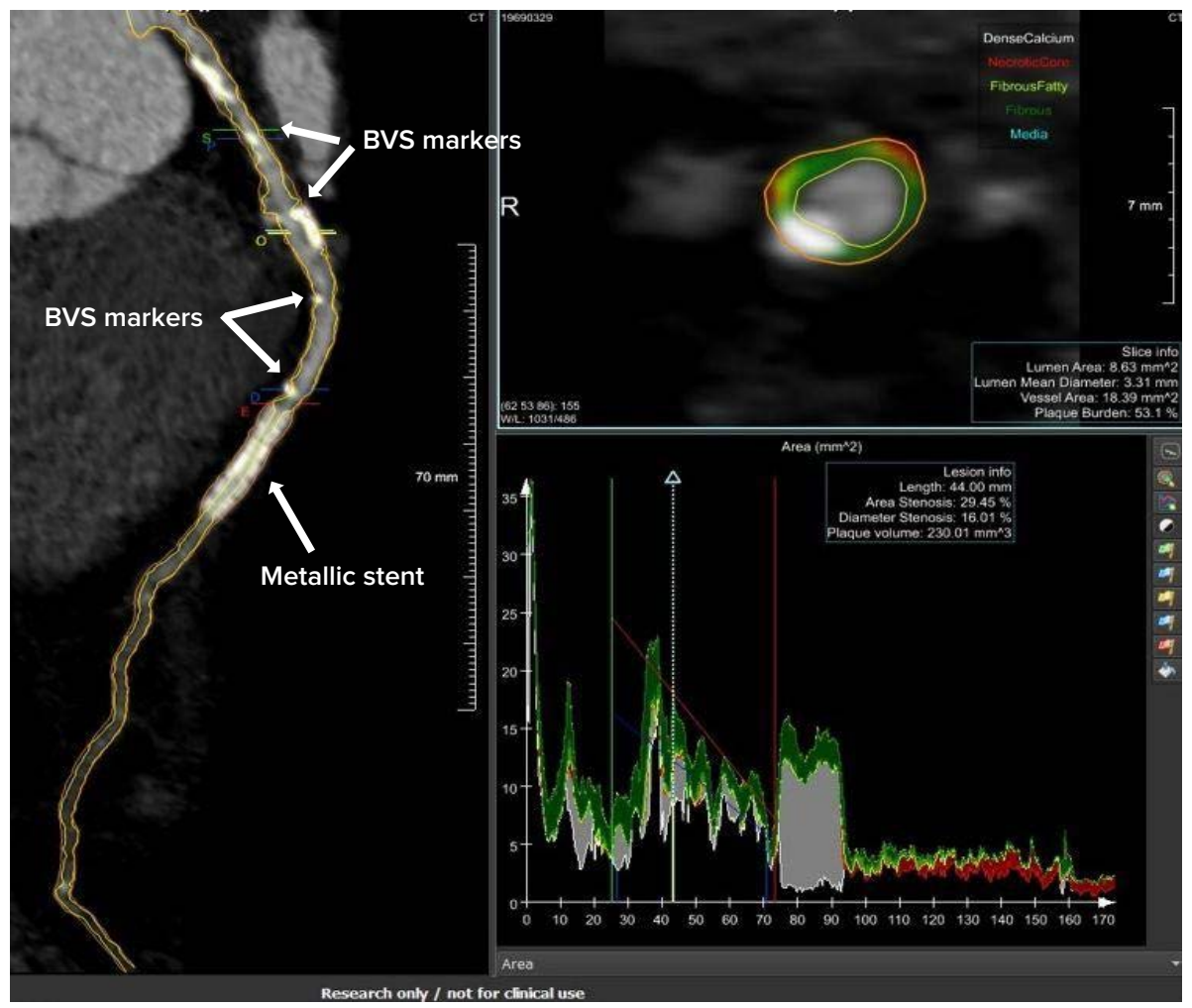

FIGURE 1. CTA of the left anterior descending artery at 12 months after PTCA with 3 BVS and 1 DES - QAngio-CT RE post-processing software (Medis, Leiden, the Netherlands)

\section{RESULTS}

\section{General characteristics of the study population}

General characteristics and the main cardiovascular risk factors in the two study groups are summarized in Table 1.

We found no significant differences regarding mean age in the study groups (Group $1-65 \pm 7$ years vs. Group $2-$ $58.07 \pm 7.75$ years, $\mathrm{p}=0.71)$.
With regard to the incidence of cardiovascular risk factors, there were no statistically significant differences regarding the prevalence of diabetes mellitus (Group $1-38 \%$ vs. Group $2-20 \%, \mathrm{p}=0.56$ ), male gender (Group $1-82 \%$ vs. Group $2-86.66 \%, \mathrm{p}=0.56$ ), or chronic tobacco use (Group $1-40 \%$ vs. Group $2-43.33 \%$, p = 1.0).

However, dyslipidemia had a higher incidence among patients from Group 1 (Group $1-73 \%$ vs. Group $2-30 \%$, $\mathrm{p}<0.0001)$.

TABLE 1. General characteristics and cardiovascular risk factors

\begin{tabular}{lccccc}
\hline & BMS, $\mathbf{n}$ & BMS, $\%$ & BVS, $\mathbf{~}$ & BVS, \% & p value \\
\hline Age (years) & $65 \pm 7$ & - & $58.07 \pm 7.75$ & - & 0.71 \\
Male gender & 37 & $82 \%$ & 26 & $86.66 \%$ & 0.56 \\
Body mass index $\left(\mathrm{kg} / \mathrm{m}^{2}\right)$ & $23.7 \pm 2.0$ & - & $24.5 \pm 2.3$ & - & 0.78 \\
Diabetes mellitus & 17 & $38 \%$ & 6 & $20 \%$ & 0.56 \\
Dyslipidemia & 33 & $73 \%$ & 9 & $30 \%$ & $<0.0001$ \\
Chronic tobacco use & 18 & $40 \%$ & 13 & $43.33 \%$ & 1.0 \\
Diseased vessels & & & & & 0.0051 \\
1 vessel & 26 & $60.46 \%$ & 13 & $43.33 \%$ & 0.0051 \\
2 vessels & 12 & $27.9 \%$ & 8 & $26,66 \%$ & 0.0051 \\
3 vessels & 5 & $11.62 \%$ & 9 & $30 \%$ & \\
\hline
\end{tabular}


TABLE 2. Stent type and characteristics

\begin{tabular}{lccc}
\hline Model & Stent material & $\begin{array}{c}\text { Strut thickness } \\
(\boldsymbol{\mu} \mathbf{m})\end{array}$ & Stent, $\mathbf{n}$ \\
\hline BMS & & & \\
MULTI-LINK VISION/Abbott Vascular & Cobalt & 81.28 & 2 \\
Resolute Integrity DES/Medtronic & Cobalt & 91.44 & 11 \\
XIENCE PRO DES Everolimus/Abbott Vascular & Cobalt & 81.28 & 28 \\
Tsunami Gold BMS/Terumo Europe & Stainless steel & 78.74 & 13 \\
MULTI-LINK PLUS/Guidant & Stainless steel & 55.88 & 9 \\
BVS & & & 50 \\
ABSORB Bioresorbable Vascular Scaffold 1.1, & Poly-L-lactic acid & 150 & \\
Abbott Vascular, Santa Clara, California & & & \\
\hline
\end{tabular}

In terms of CAD severity, Group 1 presented a significantly higher incidence of severe atherosclerotic involvement (Group $1-11.62 \%$ vs. Group $2-30 \%$, p <0.0051).

\section{Characteristics of the implanted stents}

The type and main characteristics of implanted stents are presented in Table 2.

Revascularized coronary segments with metallic and bioresorbable scaffolds are presented in Table 3.

Regarding the revascularized coronary segment, the proximal part of the right coronary artery (RCA) presented a significantly higher percentage of metallic stents compared to BVS (14\% BMS vs. $2 \%$ BVS, $p=0.0029)$. The middle (9.52\% BMS vs. $10 \%$ BVS, $p=1.0)$ and distal parts of the RCA (9.52\% BMS vs. $4 \%$ BVS, $p=0.25)$ presented no significant differences in terms of the type of implanted device.

\section{CTA performance in the evaluation of BVS vs. BMS}

The comparative analysis of CTA sensitivity in terms of visual evaluation of ISR in the two study groups identified a significantly higher percentage of diagnostic CT evaluations in the BVS group (Group $1-94 \%$ vs. Group $2-$ $76.19 \%, p=0.0006)$. The diagnostic sensitivity analysis of CTA in ISR is presented in Table 4.

Plaque analysis was possible only in the BVS group with a post-processing research-dedicated software, Syngo.via Frontier, which identified the vulnerability markers presented in Table 5.

Patients from Group 1 presented in a greater percentage $1(38.29 \%)$ or $2(25.53 \%)$ vulnerability markers (Figure 2 ).

Morphological and virtual histology aspects of the analyzed coronary plaques in the BVS group are presented in Table 6.

TABLE 3. Stent location

\begin{tabular}{lccccc}
\hline Segment & BMS, $\mathbf{n}$ & BMS, $\%$ & BVS, $\mathbf{n}$ & BVS, \% & p value \\
\hline RCA proximal & 9 & 14.29 & 1 & 2 & 0.0029 \\
RCA mid & 6 & 9.52 & 5 & 10 & 1.0 \\
RCA distal & 6 & 9.52 & 2 & 4 & 0.25 \\
LAD proximal & 9 & 14.29 & 11 & 22 & 0.66 \\
LAD mid & 14 & 22.22 & 13 & 26 & 0.61 \\
LAD apical & 1 & 1.59 & 2 & 4 & 0.36 \\
LCX proximal & 3 & 4.76 & 4 & 8 & 0.37 \\
LCX mid & 3 & 4.76 & 6 & 12 & 0.06 \\
D1 proximal & 6 & 9.52 & 3 & 6 & 0.59 \\
D1 mid & 0 & 0 & 1 & 2 & - \\
MO1 proximal & 3 & 4.76 & 0 & 0 & - \\
MO2 mid & 3 & 4.76 & 0 & 0 & - \\
Intermediate & 0 & 0 & 1 & 2 & - \\
Left main & 0 & 0 & 1 & 2 & - \\
TOTAL & 63 & 100 & 50 & 100 & \\
\hline
\end{tabular}


TABLE 4. ISR detection sensitivity of CTA

\begin{tabular}{lccccc}
\hline & \multicolumn{2}{c}{ Diagnostic CT } & \multicolumn{2}{c}{ Non-diagnostic CT } & \multirow{2}{*}{ p value } \\
\cline { 2 - 4 } & $\mathbf{n}$ & $\%$ & $\mathbf{n}$ & $\%$ & \\
\hline BVS & 47 & 94 & 3 & 6 & 0.0006 \\
BMS & 48 & 76.19 & 15 & 23.8 & 0.0006 \\
\hline
\end{tabular}

TABLE 5. CTA features of vulnerable plaques

\begin{tabular}{lc}
\hline & $\%$ \\
\hline Positive remodeling & 46.8 \\
Spotty calcification & 65.95 \\
Napkin-ring & 4.25 \\
Low attenuation & 19.14 \\
\hline
\end{tabular}

In terms of significant hemodynamic ISR, CTA evaluation provides the most accurate results for the 3.0 and $3.5 \mathrm{~mm}$ devices. In 2.5 and $4.0 \mathrm{~mm}$ devices, the number of significant ISR diagnoses was zero. Regarding the sensitivity of CTA for ISR identification, the BVS group presented the smallest incidence of non-diagnostic CT evaluations (Figure 3).

\section{DISCUSSIONS}

Following the feasibility of CTA in diagnosing ISR in BMS vs. BVS as a primary endpoint, this study demonstrated a significant difference in the specificity of this imaging

TABLE 6. Plaque morphology and virtual histology composition - Group 1

\begin{tabular}{lc}
\hline & Mean value \pm SD \\
\hline Plaque length (mm) & $17.45 \pm 4.97$ \\
Stenosis (\%) & $22.37 \pm 12.73$ \\
Eccentricity index & $0.43 \pm 0.26$ \\
Remodeling index & $1.03 \pm 0.29$ \\
Vascular volume $\left(\mathrm{mm}^{3}\right)$ & $273.2 \pm 87.42$ \\
Lumen volume $\left(\mathrm{mm}^{3}\right)$ & $119.8 \pm 46.45$ \\
Plaque volume $\left(\mathrm{mm}^{3}\right)$ & $153.6 \pm 65.0$ \\
Dense calcium ( $\left.\mathrm{mm}^{3}\right)$ & $17.48 \pm 20.43$ \\
Dense calcium $(\%)$ & $10.55 \pm 11.92$ \\
Necrotic core $\left(\mathrm{mm}^{3}\right)$ & $136.4 \pm 52.76$ \\
Necrotic core $(\%)$ & $89.45 \pm 11.92$ \\
Fibro-fatty (mm $\left.{ }^{3}\right)$ & $11.07 \pm 10.38$ \\
Fibro-fatty $(\%)$ & $6.83 \pm 5.66$ \\
Fibrous (mm $\left.{ }^{3}\right)$ & $125.1 \pm 48.53$ \\
Fibrous (\%) & $82.56 \pm 12.63$ \\
Calcium score - local & $73.79 \pm 80.19$ \\
Calcium score - target coronary artery & $186.9 \pm 207.5$ \\
\hline
\end{tabular}

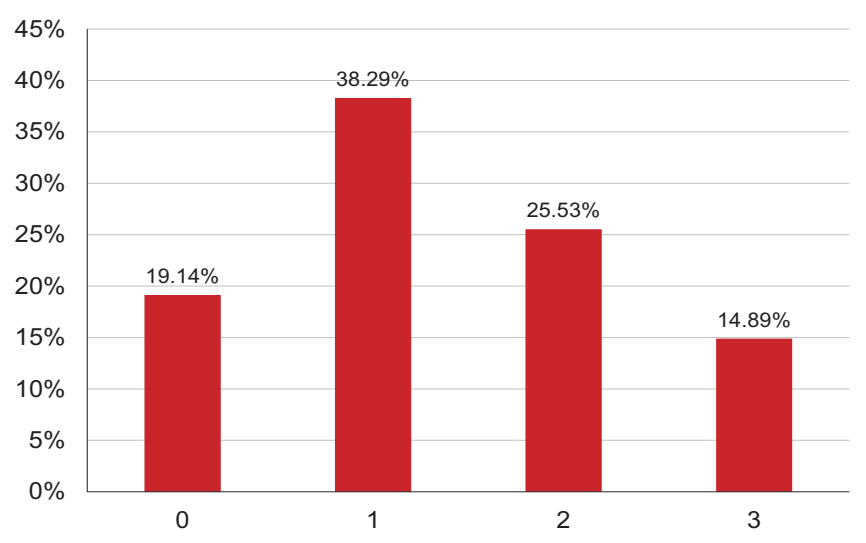

FIGURE 2. Incidence of CTA markers of vulnerability

method, showing a higher accuracy for bioresorbable scaffolds compared with metallic coronary stents. The major advantage of BVS derives from its radiolucency properties. Moreover, the inclusion of the bioresorbable matrix in the vascular wall at 12-24 months after PTCA results in the loss of the initial mechanical resistance and lumen remodeling, with an increase in size. ${ }^{16}$ Regarding metallic stents, it is a well-known fact that ISR is more common in small-diameter devices, the more so as the metallic struts are greater.

These findings are in line with data published by Oncel et al., which has shown that the visual CT analysis of 39 coronary stents, based on two independent radiologists, had a sensitivity of $89 \%$, a specificity of $95 \%$, and a positive predictive value of $90 \%$ compared to standard invasive angiography, these values being even much lower in case of devices with diameters below $2.75 \mathrm{~mm} .{ }^{17} \mathrm{In}$ a study conducted on 25 patients revascularized with metallic stents of 2.5 and $3.0 \mathrm{~mm}$ diameter, Rist et al. have shown that in this class of patients, the follow-up evaluation via CTA failed to detect the presence of asymptomatic intrastent occlusion in $17 \%$ of the cases. ${ }^{18}$ However, another study, led by Graaf et al., using 320-slice CTA evaluation in 53 patients with 89 metallic stents in vessels with diameter under $3 \mathrm{~mm}$ and strut dimensions above 140 microns, demonstrated a reduced specificity of this method in assessing the incidence of ISR, due to significant reduction of image quality through metal artifacts. ${ }^{19}$

The most common causes for reconstruction errors in the CTA evaluation of metallic stents include the following: heart rate $>65 \mathrm{bpm}$, which results in a larger number of cardiac cycles, a higher dose of irradiation in a time unit, and a loss of optimal spatial resolution due to motion artifacts, requiring the extension of acquisition over a larger number of cardiac cycles; ${ }^{20,21}$ the so-called "blooming effect" of contrast reflection, which is more evident in small 


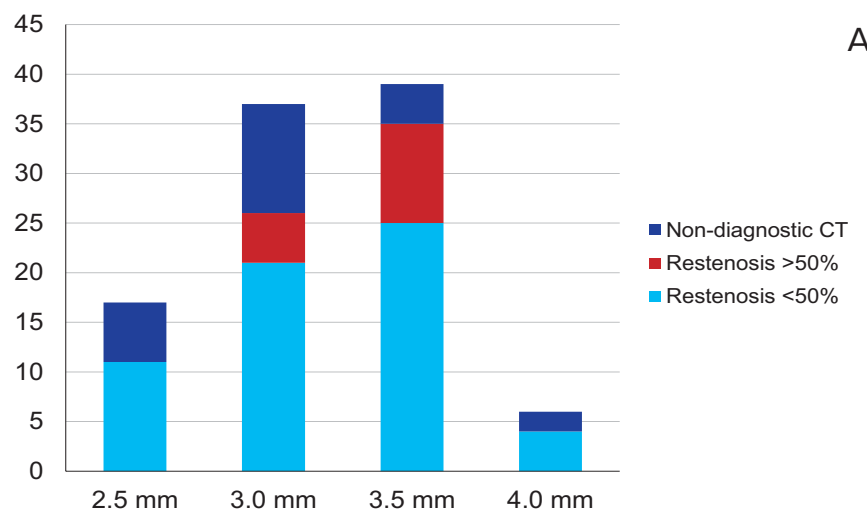

A

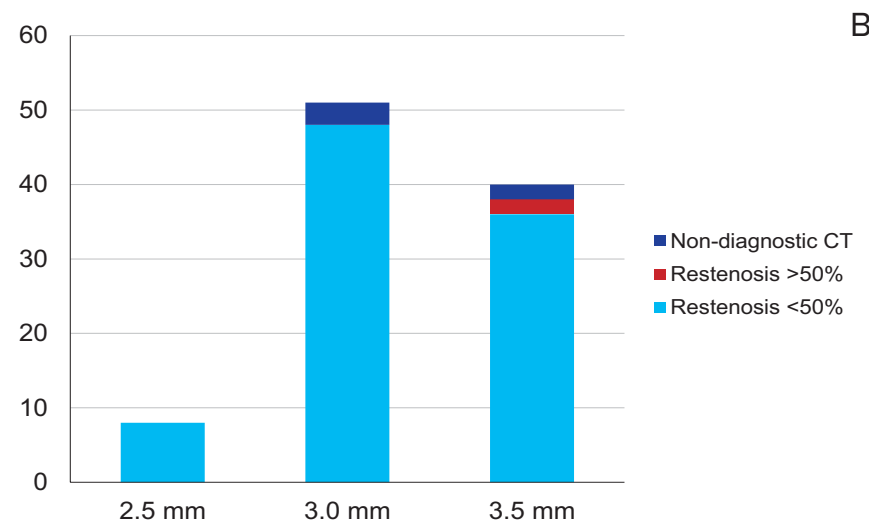

FIGURE 3. A - ISR detection sensitivity of CTA in BMS of different dimensions; B - ISR detection sensitivity of CTA in BVS of different dimensions

diameter and large strut stents and is dimmed in stents with diameter above $3 \mathrm{~mm} ;{ }^{22,23}$ stent composition: gold or gold-coated stents, as well as tantalum stents involve rough artifacts compared with steel or cobalt metallic stents. ${ }^{20}$ Proximal stent distribution on LDA, LCX, or RCA allows a better visualization of contrast material in the lumen of the stent compared to more distal segments such as diagonal or obtuse marginal branches and distal LCX. ${ }^{23}$

A series of these technical obstacles disappear in bioresorbable scaffold reconstructions. Moreover, their diameter increases at 12-24 months after PTCA due to vascular remodeling as a result of matrix resorption and loss of rigidity, characteristic for these devices.

\section{CONCLUSIONS}

The CTA evaluation of bioresorbable scaffolds is superior to metallic stent assessment, the latter being influenced by numerous sources of error dependent mainly on the presence of the metal structure. Noninvasive CT assessment is a fast and effective method compared to conventional invasive coronary evaluation, which is not a suitable screening technique due to the risks associated with the invasive nature of the procedure and the additional costs related to the need for post-interventional hospitalization for at least 24 hours.

\section{CONFLICT OF INTEREST}

Nothing to declare.

\section{ACKNOWLEDGEMENT}

This research was supported via the research grant no. 103544/2016, contract number 26/01.09.2016, entitled
"Increasing the research capacity in the field of vulnerable plaque imaging, based on advanced nanoparticles, fusion imaging and computational simulation - PlaqueImage", financed by the Romanian Ministry of European Funds, the Romanian Government and the European Union.

\section{REFERENCES}

1. Windecker S, Kolh P, Alfonso F, et al. 2014 ESC/EACTS Guidelines on myocardial revascularization. The Task Force on Myocardial Revascularization of the European Society of Cardiology (ESC) and the European Association for Cardio-Thoracic Surgery (EACTS). Eur Heart J. 2014;35:2541-2619

2. Ferent I, Mester A, Hlinomaz $\mathrm{O}$, et al. Intracoronary Imaging for Assessment of Vascular Healing and Stent Follow-up in Bioresorbable Vascular Scaffolds. Current Medical Imaging Reviews. 2018. [Epub Ahead of Print.] doi : 10.2174/1573405614666180604093621.

3. Gruntzig AR, Senning A, Siegenthaler WE. Nonoperative dilatation of coronary-artery stenosis: percutaneous transluminal coronary angioplasty. N Engl J Med. 1979;301:61-68.

4. Sigwart U, Urban P, Golf S, et al. Emergency stenting for acute occlusion after coronary balloon angioplasty. Circulation. 1988;78:1121-1127.

5. Roubin GS, Cannon AD, Agrawal SK, et al. Intracoronary stenting for acute and threatened closure complicating percutaneous transluminal coronary angioplasty. Circulation. 1992;85:916-927.

6. Benedek I, Benedek T. Angio computer tomografie multislice în diagnosticul bolilor cardiovasculare. Oradea: Editura Universității, 2014.

7. Raber L, Brugaletta S, Yamaji K, et al. Very late scaffold thrombosis: intracoronary imaging and histopathological and spectroscopic findings. $J$ Am Coll Cardiol. 2015;66:1901-1914.

8. Gao R, Yang Y, Han Y, et al. Bioresorbable vascular scaffolds versus metallic stents in patients with coronary artery disease: ABSORB China Trial. J Am Coll Cardiol. 2015;66:2298-2309.

9. Lemos PA, Serruys PW, van Domburg RT, et al. Unrestricted Utilization of Serolimus-Elutiing Stents Compared With Conventional Bare Stent Implantation in the "Real World": The Rapamycin-Eluting Stent Evaluated At Rotterdam Cardiology Hospital (RESEARCH) Registry. Circulation. 2004;109:190-195.

10. Kate FM, Morris SD, Smith HR, Rycroft RJ. Allergy in coronary in-stent restenosis. Lancet. 2001;357:1205-1206.

11. Benedek T, Bucur O, Pascanu I, Benedek I. Analysis of coronary plaque morphology by 64 multislice computed tomography coronary angiography and calcium scoring in patients with type 2 diabetes mellitus. Acta Endocrinologica. 2011;7:59-68.

12. Benedek I, Chitu M, Kovacs I, Bajka B, Benedek T. Incremental Value of preprocedural Coronary Computed Tomographic Angyography to classical Coronary Angiography for prediction of $\mathrm{PCl}$ complexity in left main stenosis. World Journal of Cardiovascular Disease. 2013;9:573-580. 
13. Benedek T, Jako B, Benedek I. Plaque quantification by coronary CT and intravascular ultrasound identifies a low CT density core as a marker of plaque instability in acute coronary syndromes. Int Heart J. 2014:55:22-28.

14. Benedek T, Gyongyosi M, Benedek I. Multislice computed tomographic coronary angiography for quantitative assessment of culprit lesions in acute coronary syndromes. Can J Cardiol. 2013;29:364-371.

15. Collet C, Chevalier B, Cequier A, et al. Diagnostic Accuracy of Coronary CT Angiography for the Evaluation of Bioresorbable Vascular Scaffolds. JACC Cardiovasc Imaging. 2018;11:722-732.

16. Chien S. Mechanotransduction and endothelial cell homeostasis: the wisdom of the cell. Am J Physiol Heart Circ Physiol. 2007;292:H1209-H1224.

17. Oncel D, Oncel G, Karaca M. Coronary stent patency and in-stent restenosis: determination with 64-section multidetector CT coronary angiography-initial experience. Radiology. 2007;242:403-409.

18. Rist C, von Ziegler F, Nikolaou K, et al. Assessment of coronary artery stent patency and restenosis using 64-slice computed tomography. Academic Radiology. 2006;13:1465-1473.

19. De Graaf FR, Schuijf JD, Van Velzen JE, et al. Diagnostic accuracy of 320-row multidetector computed tomography coronary angiography to noninvasively assess in-stent restenosis. Invest Radiol. 2010;45:331-340.

20. Pelberg R. Basic principles in Computed Tomography (CT). Cardiac CT angiography manual. London: Springer, 2015.

21. Dewey M, Zimmermann E, Deissenrieder F, et al. Noninvasive coronary angiography by 320-row computed tomography with lower radiation exposure and maintained diagnostic accuracy: comparison of results with cardiac catheterization in a head-to-head pilot investigation. Circulation. 2009;120:867-875.

22. Carbone I, Francone M, Algeri E, et al. Non-invasive evaluation of coronary artery stent patency with retrospectively ECG-gated 64-slice CT angiography. Eur Radiol. 2008;18:234-243.

23. Dawoud MA, Alarabawy RA, Abd Alla TM, Mubarak AA. Evaluation of coronary stents using multidetector CT. The Egyptian Journal of Radiology and Nuclear Medicine. 2016;47:793-801.

24. Yoshimura M, Nao T, Miura T, et al. New quantitative method to diagnose coronary in-stent restenosis by 64 -multislice computed tomography. $J$ Cardiol. 2015;65:57-62 